\title{
Synthesis, characterization and bio-activity of metal complexes of bidentate N-S isomeric Schiff bases derived from S-methyldithiocarbazate (SMDTC) and the X-ray structure of the bis[S-methyl- $\beta$-N-(2-furyl-methylketone) dithiocarbazato]cadmium(II) complex.
}

\begin{abstract}
Condensation of SMDTC with 2-furyl-methylketone and 5-methyl-2-furaldehyde gave isomeric Schiff bases, (NS) and (NS'). The metal complexes of these uninegatively charged bidentate Schiff base ligands with cadmium, tin, iron, lead and cobalt have been prepared. The complexes have been characterized by a variety of physico-chemical techniques. X-ray crystallographic analysis shows that the $\mathrm{Cd}(\mathrm{II})$ complex, bis[S-methyl- $\beta-\mathrm{N}-(2-$ furylmethylketone)dithiocarbazato]cadmium(II), consists of two molecules with distorted octahedral structure. The $\mathrm{Co}$ (II) complexes are paramagnetic with a square-planar stereochemistry. The diamagnetic $\mathrm{Sn}(\mathrm{II}), \mathrm{Fe}(\mathrm{III})$ and $\mathrm{Pb}(\mathrm{II})$ complexes also have squareplanar structures while the diamagnetic [Cd(NS')2]. 3H2O complex is tetrahedral. The [Cd(NS')2].3H2O, [Sn(N'S)2) and [Co(NS)2] showed clear inhibition of almost all bacteria and fungi tested However, [Cd(NS)2], [Fe(NS)]Cl2, [Pb(NS)2] and [Pb(NS')2] were inactive against all bacteria assayed while [Cd(NS)2], [Fe(NS)]Cl2 and $[\mathrm{Pb}(\mathrm{NS}) 2]$ showed clear inhibition of some fungi. The [Cd(NS)2], [Cd(NS')2].3H2O and [Co(NS)2] were found to be very active against Human cell T-lymphoblastic leukemia (CEM-SS) and cervical cancer cells (HELA) with CD50 values between 1.8 and $3.6 \mu 1 \mathrm{~cm}-3$.
\end{abstract}

Keyword: Dithiocarbazate; Schiff base; 2-furyl-methylketone; Cadmium(II); X-ray structure. 